\section{Lur'e Lyapunov Function and Absolute Stability Criterion for Lur'e Singularly Perturbed Systems}

Chunyu Yang, Member, IEEE, Qingling Zhang,

Jing Sun, Fellow, IEEE, and Tianyou Chai, Fellow, IEEE

\begin{abstract}
This technical note investigates the absolute stability problem for Lur'e singularly perturbed systems with multiple nonlinearities. The objective is to determine if the system is absolutely stable for any $\varepsilon \in\left(0, \varepsilon_{0}\right]$, where $\varepsilon$ denotes the perturbation parameter and $\varepsilon_{0}$ is a pre-defined positive scalar. First, an $\varepsilon$-dependent Lur'e Lyapunov function is constructed that facilitates the stability analysis of the singularly perturbed system. Then, a stability criterion expressed in terms of $\varepsilon$-independent linear matrix inequalities (LMIs) is derived. Based on the stability criterion, an algorithm is proposed to compute the stability bound that is shown to be less conservative than those computed using other existing methods. Finally, examples are given to show the feasibility and effectiveness of the obtained method.
\end{abstract}

Index Terms-Absolute stability, linear matrix inequality (LMI), Lur'e Lyapunov function, Lur'e singularly perturbed systems (SPSs).

\section{INTRODUCTION}

Many dynamical systems, such as convection-diffusion systems, power systems, magnetic-ball suspension systems, contain both slow and fast dynamical phenomena which may lead to high dimensionality and ill-conditioned issues in system analysis and controller design problems. To deal with these problems, the multiple-time-scale systems are usually modeled as singularly perturbed systems (SPSs), with a small singular perturbation parameter $\varepsilon$ determining the degree of separation between the "slow" and "fast" modes of the system (see, e.g., [1]-[12]). For SPSs, a key aspect is analyzing the robustness of system stability/performance with respect to the perturbation parameter $\varepsilon$. The robustness of stability is often referred to as the problem of determining the stability bound $\varepsilon_{0}$ such that the system is stable for all $\varepsilon \in\left(0, \varepsilon_{0}\right)$ or $\left(0, \varepsilon_{0}\right]$. Many results on the stability bound problem of SPSs have been developed (see, e.g., [1]-[5]).

This technical note will consider the stability bound problem of the following Lur'e SPS:

$$
\left\{\begin{array}{l}
\dot{x}_{1}=A_{11} x_{1}+A_{12} x_{2}+B_{1} \phi(\sigma) \\
\varepsilon \dot{x}_{2}=A_{21} x_{1}+A_{22} x_{2}+B_{2} \phi(\sigma) \\
\sigma=C_{1} x_{1}+C_{2} x_{2}
\end{array}\right.
$$

where $x_{i} \in R^{n_{i}},(i=1,2)$ denote the state, $A_{i j}, B_{i}, C_{i},(i, j=1,2)$ are constant real matrices with appropriate dimensions, $\phi(\sigma) \in R^{m}$ is

Manuscript received November 23, 2010; revised March 14, 2011; accepted May 20, 2011. Date of publication May 31, 2011; date of current version November 02, 2011. This technical note was presented in part at the American Control Conference, Baltimore, MD, 2010. This work was supported by the Natural Science Foundation of China (60904009, 60904079, 60974004, 61020106003), the Fundamental Research Funds for the Central Universities (N090408001), the National Basic Research Program of China (2009CB320601), the Funds for Creative Research Groups of China (60821063), and the 111 project (B08015). Recommended by Associate Editor P. Tabuada.

C. Yang and T. Chai are with the State Key Laboratory of Integrated Automation for Process Industries, Northeastern University, Shenyang 110819, China (e-mail: chunyuyang@yahoo.cn; tychai@mail.neu.edu.cn).

Q. Zhang is with the Institute of Systems Science, Northeastern University, Shenyang 110819, China (e-mail: qlzhang@mail.neu.edu.cn; qlzhangneu1@163.com).

J. Sun is with the Department of Electrical Engineering and Computer Science, University of Michigan, Ann Arbor, MI 48109 USA (e-mail: jingsun@umich.edu).

Digital Object Identifier 10.1109/TAC.2011.2158136 a continuous function of $\sigma \in R^{m}$ and satisfies the sector condition

$$
\phi^{T}(\sigma)(\phi(\sigma)-K \sigma) \leq 0, \forall \sigma \in R^{m}
$$

or, equivalently

$$
0 \leq \frac{\phi_{j}\left(\sigma_{j}\right)}{\sigma_{j}} \leq k_{j}, j=1,2, \ldots, m
$$

where $K=\operatorname{diag}\left(k_{1}, k_{2}, \ldots, k_{m}\right)>0, \sigma=\left[\begin{array}{llll}\sigma_{1} & \sigma_{2} & \cdots & \sigma_{m}\end{array}\right]^{T}$, $\phi(\sigma)=\left[\begin{array}{llll}\phi_{1}\left(\sigma_{1}\right) & \phi_{2}\left(\sigma_{2}\right) & \cdots & \phi_{m}\left(\sigma_{m}\right)\end{array}\right]^{T}$.

For a given $\varepsilon$,Lur'e SPS (1) is said to be absolutely stable if it is globally asymptotically stable for any $\phi(\sigma)$ satisfying (2). It is well-known that absolute stability of Lur'e systems is a fundamental problem of control theory and plays a key role in many design problems of automatic control systems. For normal systems, absolute stability has been widely studied and many time- and frequency-domain criteria have been reported [13], [14]. Since a direct application of these methods to Lur'e SPSs may lead to ill-conditioned issues, some efforts have been made to generalize the classical absolute stability criteria. Lur'e SPSs with sole nonlinearity are considered in [15]. Under the assumption that the reduced-order slow subsystem is absolutely stable, it is shown that the original system is absolutely stable for sufficiently small $\varepsilon$. A limitation of these results is that they require the nonlinearity to be dependent only on the slow dynamics. In [5], Lur'e SPSs with multiple nonlinearities dependent on both the slow and fast dynamics are considered. Some frequency-domain conditions for the existence of stability bound for Lur'e SPSs are derived, but the value of the stability bound is not given.

In this technical note, the absolute stability of Lur'e SPSs with multiple nonlinearities is considered. We pre-define an upper bound $\varepsilon_{0}$ for the perturbation parameter $\varepsilon$ and try to determine if the system is absolutely stable for all $\varepsilon \in\left(0, \varepsilon_{0}\right]$. To deal with this problem, we construct an $\varepsilon$-dependent Lur'e Lyapunov function by which an LMI version of stability criterion is derived. Compared with the existing results, the newly developed method has the following advantages: (1) it addresses a more general class of systems, where the nonlinear terms depend on both the fast and slow dynamics; (2) not only the existence of the stability bound is established, but also an estimate of the stability bound $\varepsilon_{0}$ is given; (3) an constructive algorithm for computing the best estimate of the stability bound is proposed.

The rest of this technical note is organized as follows. In Section II, the problem under consideration is formulated. The main results are given in Section III. In Section IV, two examples are given to show the effectiveness and advantages of the proposed methods. Section V concludes this technical note.

\section{PRoblem Formulation}

System (1) can be written as the following compact form:

$$
\left\{\begin{array}{l}
E(\varepsilon) \dot{x}=A x+B \phi(\sigma), \\
\sigma=C x
\end{array}\right.
$$

where $x=\left[\begin{array}{l}x_{1} \\ x_{2}\end{array}\right] \in R^{n}, E(\varepsilon)=\left[\begin{array}{cc}I & 0 \\ 0 & \varepsilon I\end{array}\right], B=\left[\begin{array}{c}B_{1} \\ B_{2}\end{array}\right], A=$ $\left[\begin{array}{ll}A_{11} & A_{12} \\ A_{21} & A_{22}\end{array}\right], C=\left[\begin{array}{ll}C_{1} & C_{2}\end{array}\right]$.

Under the assumption that $A_{22}$ is nonsingular, [5] decomposed system (1) into two reduced-order subsystems and showed that the circle criterion for the slow system and the Popov criterion for the fast system are sufficient for absolute stability of Lur'e SPS (1) as long as the perturbation parameter $\varepsilon$ is sufficiently small. This technical note tries to present an estimate of the stability bound for Lur'e SPS (1). Thus we define the following problem.

1) Problem 1: Given a scalar $\varepsilon_{0}>0$, determine if system (1) is absolutely stable for any $\varepsilon \in\left(0, \varepsilon_{0}\right]$. 
Remark 2.1: When $\varepsilon=1$, system (1) becomes a normal system and its absolute stability problem has been extensively investigated [13], [14]. When $\varepsilon=0$, system (1) becomes a Lur'e descriptor system and the absolute stability has been recently developed in [16], [17]. Hence, Problem 1 is complementary to the absolute stability problems of normal systems and descriptor systems.

\section{MAIN RESULTS}

We will first construct an $\varepsilon$-dependent Lur'e Lyapunov function and then derive an LMI version of stability criterion.

\section{A. A Lur'e Lyapunov Function for Lur'e SPSs}

Lyapunov functions play a predominant role in stability analysis and control for dynamic systems. Because of the two time-scale property, Lyapunov functions for SPSs are more complex than those for normal systems. A major class of Lyapunov functions for SPSs are composed as a weighted sum of the Lyapunov functions of the reduced and boundary-layer systems [9]. Another type of Lyapunov functions for SPSs depend on the perturbation parameter $\varepsilon$. Several classes of $\varepsilon$-dependent Lyapunov functions have been discussed in the literature. In [11], [12], [18], different classes of quadratic $\varepsilon$-dependent Lyapunov functions have been constructed. The newest one is given by [18] and shown in the following

$$
V(x)=x^{T}\left[\begin{array}{cc}
Z_{1}+\varepsilon Z_{3} & \varepsilon Z_{5}^{T} \\
\varepsilon Z_{5} & \varepsilon Z_{2}+\varepsilon^{2} Z_{4}
\end{array}\right] x
$$

where $Z_{i}(i=1,2, \ldots, 5)$ are constant matrices with appropriate dimensions and $Z_{i}=Z_{i}^{T}(i=1,2,3,4)$. Under certain conditions, Lyapunov function (5) is positive definite. The quadratic $\varepsilon$-dependent Lyapunov functions used by [11] and [12] are special cases of (5) with $Z_{3}=0, Z_{4}=0$.

In this subsection, we will construct an $\varepsilon$-dependent Lur'e Lyapunov function to solve Problem 1. To do this, let

$$
C=\left[\begin{array}{c}
c^{1} \\
c^{2} \\
\vdots \\
c^{m}
\end{array}\right], C_{i}=\left[\begin{array}{c}
c_{i}^{1} \\
c_{i}^{2} \\
\vdots \\
c_{i}^{m}
\end{array}\right], i=1,2
$$

then $c^{j}=\left[\begin{array}{ll}c_{1}^{j} & c_{2}^{j}\end{array}\right], \sigma_{j}=c^{j} x, j=1,2, \ldots, m$.

Let

$$
Z(\varepsilon)=\left[\begin{array}{cc}
Z_{1}+\varepsilon Z_{3} & \varepsilon Z_{5}^{T} \\
Z_{5} & Z_{2}+\varepsilon Z_{4}
\end{array}\right]
$$

where $Z_{i}(i=1,2, \ldots, 5)$ are constant matrices with appropriate dimensions and $Z_{i}=Z_{i}^{T}(i=1,2,3,4)$.

Define an $\varepsilon$-dependent Lur'e Lyapunov function

$$
V(x)=x^{T} E(\varepsilon) Z(\varepsilon) x+2 \sum_{j=1}^{m} \int_{0}^{\sigma_{j}} \lambda_{j} \alpha_{j} \phi_{j}(s) d s
$$

where $\lambda_{j}, j=1,2, \ldots, m$ are design parameters pertaining to the choice of a Lyapunov function and chosen to satisfy

$$
\begin{cases}\lambda_{j}=0, & \text { if } c_{1}^{j} \neq 0 \text { and } c_{2}^{j} \neq 0, \\ \lambda_{j} \in R, & \text { otherwise }\end{cases}
$$

and $\alpha_{j}, j=1,2, \ldots, m$ are defined by

$$
\begin{cases}\alpha_{j}=1, & \text { if } c_{2}^{j}=0 \text { and } c_{1}^{j} \neq 0, \\ \alpha_{j}=\varepsilon, & \text { if } c_{1}^{j}=0 \text { and } c_{2}^{j} \neq 0, \\ \alpha_{j}=0, & \text { otherwise. }\end{cases}
$$

Remark 3.1: In (7), $\lambda_{j}$ are design parameters to be chosen so that (7) can serve as a Lyapunov function. $\alpha_{j}$ are fixed for a given system in the form of (1), where $c_{1}^{j}$ and $c_{2}^{j}$ are defined. Such a definition for $\alpha_{j}$ aims to provide an easy way to calculate the derivative of the integral terms of (7) along the trajectories of system (1).

Note that $E(\varepsilon) Z(\varepsilon)=Z^{T}(\varepsilon) E(\varepsilon)$ and $\lambda_{j} \in R, j=1,2, \ldots, m$. In general, $V(x)$ defined by (7) is not positive definite. We shall present a sufficient condition for Lyapunov function (7) to be positive definite and radially unbounded.

Lemma 3.1: Lyapunov function (7) is positive definite and radially unbounded for any $\varepsilon \in\left(0, \varepsilon_{0}\right]$ and $\phi$ satisfying (2) if

$$
E(\varepsilon)\left(Z(\varepsilon)+C^{T} \Lambda K_{\Delta} C\right)>0, \forall \varepsilon \in\left(0, \varepsilon_{0}\right]
$$

for any constant diagonal matrix $K_{\Delta}$ with $K_{\Delta} \in \Omega$, where $\Omega=$ $\left\{\operatorname{diag}\left(\delta_{1}, \delta_{2}, \ldots, \delta_{m}\right) \mid \forall \delta_{j} \in\left\{0, k_{j}\right\}\right\}, \Lambda=\operatorname{diag}\left\{\lambda_{1}, \lambda_{2}, \ldots, \lambda_{m}\right\}$, $k_{j}$ and $\lambda_{j}$ are defined by (3) and (8), respectively.

Proof: From the definition of $\lambda_{j}$ and $\alpha_{j}$, one can see that $\lambda_{j} \alpha_{j} c_{1}^{j}=\lambda_{j} c_{1}^{j}$ holds for all the cases of $c_{1}^{j}$ and $c_{2}^{j}$. Similarly, we have $\lambda_{j} \alpha_{j} c_{2}^{j}=\varepsilon \lambda_{j} c_{2}^{j}$. Then we can write

$$
\lambda_{j} \alpha_{j} c^{j}=\lambda_{j}\left[\begin{array}{cc}
c_{1}^{j} & \varepsilon c_{2}^{j}
\end{array}\right]=\lambda_{j} c^{j} E(\varepsilon)
$$

which shows $\Lambda C E(\varepsilon)=\Lambda \Pi C$ with $\Pi=\operatorname{diag}\left(\alpha_{1}, \alpha_{2}, \ldots, \alpha_{m}\right)$, leading to

$$
E(\varepsilon) C^{T} \Lambda K_{\Delta} C=C^{T} \Pi \Lambda K_{\Delta} C
$$

for any diagonal matrix $K_{\Delta}$. From (6) and (12), it follows that $E(\varepsilon)\left(Z(\varepsilon)+C^{T} \Lambda K_{\Delta} C\right)$ is symmetric.

Let

$$
\begin{aligned}
& I_{+} \triangleq\left\{j \mid \lambda_{j} \geq 0,1 \leq j \leq m\right\}, \\
& I_{-} \triangleq\left\{j \mid \lambda_{j}<0,1 \leq j \leq m\right\} \\
& \bar{\Lambda} \triangleq \operatorname{diag}\left(\bar{\lambda}_{1}, \bar{\lambda}_{2}, \ldots, \bar{\lambda}_{m}\right), \bar{\lambda}_{j} \triangleq \begin{cases}\lambda_{j}, & j \in I_{+}, \\
0, & j \in I_{-},\end{cases} \\
& \underline{\Lambda} \triangleq \operatorname{diag}\left(\underline{\lambda}_{1}, \underline{\lambda}_{2}, \ldots, \underline{\lambda}_{m}\right), \underline{\lambda}_{j} \triangleq \begin{cases}-\lambda_{j}, & j \in I_{-}, \\
0, & j \in I_{+},\end{cases} \\
& K_{\Delta} \triangleq \operatorname{diag}\left(k_{\Delta 1}, k_{\Delta 2}, \ldots, k_{\Delta m}\right), k_{\Delta j} \triangleq \begin{cases}k_{j}, & j \in I_{-}, \\
0, & j \in I_{+} .\end{cases}
\end{aligned}
$$

It can be seen that

$$
\bar{\Lambda} \geq 0, \underline{\Lambda} \geq 0, K_{\Delta} \in \Omega, \Lambda=\bar{\Lambda}-\underline{\Lambda}, \Lambda K_{\Delta}=-\underline{\Lambda} K .
$$

From (12), we have $E(\varepsilon) C^{T} \underline{\Lambda} K C=C^{T} \Pi \underline{\Lambda} K C$ which shows

$$
\begin{aligned}
x^{T} E(\varepsilon) C^{T} \underline{\Lambda} K C x & =x^{T} C^{T} \Pi \underline{\Lambda} K C x \\
& =\sigma^{T} \Pi \underline{\Lambda} K \sigma \\
& =\sum_{j=1}^{m} \underline{\lambda}_{j} \alpha_{j} k_{j}\left(\sigma^{j}\right)^{2} \\
& =2 \sum_{j=1}^{m} \underline{\lambda}_{j} \alpha_{j} \int_{0}^{\sigma_{j}} k_{j} s d s .
\end{aligned}
$$

Taking into account (13) and (14), we have

$$
\begin{aligned}
V(x)= & x^{T} E(\varepsilon) Z(\varepsilon) x+2 \sum_{j=1}^{m} \int_{0}^{\sigma_{j}} \lambda_{j} \alpha_{j} \phi_{j}(s) d s \\
= & x^{T} E(\varepsilon) Z(\varepsilon) x-x^{T} E(\varepsilon) C^{T} \underline{\Lambda} K C x \\
& +2 \sum_{j=1}^{m} \int_{0}^{\sigma_{j}} \lambda_{j} \alpha_{j} \phi_{j}(s) d s+x^{T} E(\varepsilon) C^{T} \underline{\Lambda} K C x \\
= & x^{T} E(\varepsilon)\left(Z(\varepsilon)+C^{T} \Lambda K_{\Delta} C\right) x \\
& +2 \sum_{j=1}^{m}\left(\bar{\lambda}_{j}-\underline{\lambda}_{j}\right) \alpha_{j} \int_{0}^{\sigma_{j}} \phi_{j}(s) d s \\
& +2 \sum_{j=1}^{m} \underline{\lambda}_{j} \alpha_{j} \int_{0}^{\sigma_{j}} k_{j} s d s
\end{aligned}
$$




$$
\begin{aligned}
= & x^{T} E(\varepsilon)\left(Z(\varepsilon)+C^{T} \Lambda K_{\Delta} C\right) x \\
& +2 \sum_{j=1}^{m} \bar{\lambda}_{j} \alpha_{j} \int_{0}^{\sigma_{j}} \phi_{j}(s) d s \\
& +2 \sum_{j=1}^{m} \underline{\lambda}_{j} \alpha_{j} \int_{0}^{\sigma_{j}}\left(k_{j} s-\phi_{j}(s)\right) d s .
\end{aligned}
$$

Sector condition (2) implies that

$$
\int_{0}^{\sigma_{j}} \phi_{j}(s) d s \geq 0 \text { and } \int_{0}^{\sigma_{j}}\left(k_{j} s-\phi_{j}(s)\right) d s \geq 0 .
$$

Then by (15), we have

$$
V(x) \geq x^{T} E(\varepsilon)\left(Z(\varepsilon)+C^{T} \Lambda K_{\Delta} C\right) x .
$$

It follows from (10) and (16) that the Lyapunov function (7) is positive definite and radially unbounded for any $\varepsilon \in\left(0, \varepsilon_{0}\right]$ and any $\phi$ satisfying (2).

Remark 3.2: In robust stability analysis and synthesis problems, Lur'e Lyapunov functions have advantages over quadratic Lyapunov functions [19], [20]. It is for the first time that a Lur'e Lyapunov function is proposed for SPSs to the authors' best knowledge. We expect that it can be used in the problems of robust stability analysis and synthesis for SPSs.

\section{B. Stability Criterion}

In this subsection, we will propose a stability criterion by using Lyapunov function (7). We first recall the following lemmas.

Lemma 3.2: [18] For a positive scalar $\varepsilon_{0}$ and symmetric matrices $S_{1}, S_{2}$ and $S_{3}$ with appropriate dimensions, if

$$
\begin{aligned}
S_{1} & \geq 0 \\
S_{1}+\varepsilon_{0} S_{2} & >0 \\
S_{1}+\varepsilon_{0} S_{2}+\varepsilon_{0}^{2} S_{3} & >0
\end{aligned}
$$

hold, then

$$
S_{1}+\varepsilon S_{2}+\varepsilon^{2} S_{3}>0, \forall \varepsilon \in\left(0, \varepsilon_{0}\right] .
$$

Lemma 3.3: [18] If there exist matrices $Z_{i}(i=1,2, \ldots, 5)$ with $Z_{i}=Z_{i}^{T}(i=1,2,3,4)$ satisfying

$$
\begin{aligned}
{\left[\begin{array}{cc}
Z_{1}+\varepsilon_{0} Z_{3} & \varepsilon_{0} Z_{5}^{T} \\
\varepsilon_{0} Z_{5} & \varepsilon_{0} Z_{2}
\end{array}\right] } & >0 \\
{\left[\begin{array}{cc}
Z_{1}+\varepsilon_{0} Z_{3} & \varepsilon_{0} Z_{5}^{T} \\
\varepsilon_{0} Z_{5} & \varepsilon_{0} Z_{2}+\varepsilon_{0}^{2} Z_{4}
\end{array}\right] } & >0
\end{aligned}
$$

then

$$
E(\varepsilon) Z(\varepsilon)=Z^{T}(\varepsilon) E(\varepsilon)>0, \forall \varepsilon \in\left(0, \varepsilon_{0}\right] .
$$

Theorem 3.1: Given a scalar $\varepsilon_{0}>0$, if there exist a diagonal matrix $\Gamma>0$, matrices $Z_{i}(i=1,2, \ldots, 5)$ with $Z_{i}=Z_{i}^{T}(i=1,2,3,4)$ and $\Lambda=\operatorname{diag}\left\{\lambda_{1}, \lambda_{2}, \ldots, \lambda_{m}\right\}$ with $\lambda_{j}=0$ when $c_{1}^{j} \neq 0$ and $c_{2}^{j} \neq$ 0 , such that LMIs (21)-(23) and

$$
\left[\begin{array}{cc}
A^{T} U_{1}+U_{1}^{T} A & \Theta_{1} \\
B^{T} U_{1}+\Lambda C A+\Gamma K C & \Lambda C B+B^{T} C^{T} \Lambda-2 \Gamma
\end{array}\right]<0
$$

$$
\left[\begin{array}{cc}
A^{T}\left(U_{1}+\varepsilon_{0} U_{2}\right) & \\
+\left(U_{1}+\varepsilon_{0} U_{2}\right)^{T} A & \Theta_{2} \\
B^{T}\left(U_{1}+\varepsilon_{0} U_{2}\right) & \\
+\Lambda C A+\Gamma K C & \Lambda C B+B^{T} C^{T} \Lambda-2 \Gamma
\end{array}\right]<0
$$

hold, where

$$
\begin{aligned}
& \Theta_{1}=U_{1}^{T} B+A^{T} C^{T} \Lambda+C^{T} K \Gamma, \\
& \Theta_{2}=\left(U_{1}+\varepsilon_{0} U_{2}\right)^{T} B+A^{T} C^{T} \Lambda+C^{T} K \Gamma, \\
& U_{1}=\left[\begin{array}{cc}
Z_{1} & 0 \\
Z_{5} & Z_{2}
\end{array}\right], U_{2}=\left[\begin{array}{cc}
Z_{3} & Z_{5}^{T} \\
0 & Z_{4}
\end{array}\right] .
\end{aligned}
$$

Then system (1) is absolutely stable for any $\varepsilon \in\left(0, \varepsilon_{0}\right]$.

Proof: Computing the derivative of $V(x)$ along the trajectories of system (4) and using (11), we have

$$
\begin{aligned}
\left.\dot{V}\right|_{(4)}= & (E(\varepsilon) \dot{x})^{T} Z(\varepsilon) x+x^{T} Z^{T}(\varepsilon) E(\varepsilon) \dot{x} \\
& +2 \sum_{j=1}^{m} \dot{\sigma}_{j} \lambda_{j} \alpha_{j} \phi_{j}\left(\sigma_{j}\right) \\
= & x^{T}\left(A^{T} Z(\varepsilon)+Z^{T}(\varepsilon) A\right) x+2 \phi^{T} B^{T} Z(\varepsilon) x \\
& +2 \sum_{j=1}^{m} \lambda_{j} c^{j} E(\varepsilon) \dot{x} \phi_{j}\left(\sigma_{j}\right) \\
= & x^{T}\left(A^{T} Z(\varepsilon)+Z^{T}(\varepsilon) A\right) x \\
& +2 \phi^{T} B^{T} Z(\varepsilon) x+2(E(\varepsilon) \dot{x})^{T} C^{T} \Lambda \phi \\
= & x^{T}\left(A^{T} Z(\varepsilon)+Z^{T}(\varepsilon) A\right) x \\
& +2 \phi^{T} B^{T} Z(\varepsilon) x+2(A x+B \phi)^{T} C^{T} \Lambda \phi \\
= & {\left[\begin{array}{l}
x \\
\phi
\end{array}\right]^{T} S\left[\begin{array}{l}
x \\
\phi
\end{array}\right] }
\end{aligned}
$$

where $S=\left[\begin{array}{cc}A^{T} Z(\varepsilon)+Z^{T}(\varepsilon) A & Z^{T}(\varepsilon) B+A^{T} C^{T} \Lambda \\ B^{T} Z(\varepsilon)+\Lambda C A & \Lambda C B+B^{T} C^{T} \Lambda\end{array}\right]$.

By (2), for any diagonal positive definite matrix $\Gamma \in R^{m}$, it holds that

$$
\phi^{T} \Gamma(\phi-K C x) \leq 0, \forall x \in R^{n} .
$$

Then we have (29), shown at the bottom of the page. By Lemma 3.2, it follows from (25) and (26) and (29) that:

$$
\left.\dot{V}\right|_{(4)}<0, \forall x \neq 0
$$

holds for any $\varepsilon \in\left(0, \varepsilon_{0}\right]$ and $\phi$ satisfying (2).

On the other hand, let $K_{\Delta}$ be a diagonal matrix and satisfy $0 \leq$ $K_{\Delta} \leq K$. Then $\phi \triangleq K_{\Delta} \sigma$ satisfies (2). In this case, from (27), one gets (31), shown at the bottom of the next page. Inequalities (30) and (31) show that

$$
\begin{aligned}
\left(A+B K_{\Delta} C\right)^{T}( & \left.Z(\varepsilon)+C^{T} \Lambda K_{\Delta} C\right) \\
& +\left(Z(\varepsilon)+C^{T} \Lambda K_{\Delta} C\right)^{T}\left(A+B K_{\Delta} C\right)<0
\end{aligned}
$$

holds for any $\varepsilon \in\left(0, \varepsilon_{0}\right]$ and diagonal matrix $K_{\Delta}$ with $0 \leq K_{\Delta} \leq K$. Then matrix $Z(\varepsilon)+C^{T} \Lambda K_{\Delta} C$ is nonsingular for any $\varepsilon \in\left(0, \varepsilon_{0}\right]$ and diagonal matrix $K_{\Delta}$ with $0 \leq K_{\Delta} \leq K$, so is $E(\varepsilon)(Z(\varepsilon)+$ $C^{T} \Lambda K_{\Delta} C$ ). Using Lemma 3.3, LMIs (21)-(23) imply $E(\varepsilon) Z(\varepsilon)>$

$$
\begin{aligned}
\left.\dot{V}\right|_{(4)} & \leq\left[\begin{array}{l}
x \\
\phi
\end{array}\right]^{T}\left[\begin{array}{cc}
A^{T} Z(\varepsilon)+Z(\varepsilon)^{T} A & Z^{T}(\varepsilon) B+A^{T} C^{T} \Lambda \\
B^{T} Z(\varepsilon)+\Lambda C A & \Lambda C B+B^{T} C^{T} \Lambda
\end{array}\right]\left[\begin{array}{l}
x \\
\phi
\end{array}\right]-2 \phi^{T} \Gamma(\phi-K C x) \\
& =\left[\begin{array}{l}
x \\
\phi
\end{array}\right]^{T}\left[\begin{array}{cc}
A^{T} Z(\varepsilon)+Z(\varepsilon)^{T} A & Z^{T}(\varepsilon) B+A^{T} C^{T} \Lambda+C^{T} K \Gamma \\
B^{T} Z(\varepsilon)+\Lambda C A+\Gamma K C & \Lambda C B+B^{T} C^{T} \Lambda-2 \Gamma
\end{array}\right]\left[\begin{array}{l}
x \\
\phi
\end{array}\right] .
\end{aligned}
$$


$0, \varepsilon \in\left(0, \varepsilon_{0}\right]$. Then, by the arbitrariness of $K_{\Delta}$ and the nonsingularity of $E(\varepsilon)\left(Z(\varepsilon)+C^{T} \Lambda K_{\Delta} C\right)$, one can conclude that $E(\varepsilon)(Z(\varepsilon)+$ $\left.C^{T} \Lambda K_{\Delta} C\right)>0$ holds for any $\varepsilon \in\left(0, \varepsilon_{0}\right]$ and diagonal matrix $K_{\Delta}$ with $0 \leq K_{\Delta} \leq K$. Then, by Lemma 3.1, Lyapunov function (7) is positive definite and radially unbounded for any $\varepsilon \in\left(0, \varepsilon_{0}\right]$ and $\phi$ satisfying (2).

Hence, it follows from (30) that system (1) is absolutely stable for any $\varepsilon \in\left(0, \varepsilon_{0}\right]$.

Remark 3.3: It is known that LMIs can be solved by numerical algorithms in polynomial time [21]. Some of these algorithms have been incorporated into different control analysis and design tools for the resolution of LMI problems [22]. LMI Toolbox in Matlab can be used to solve the LMI conditions in Theorem 3.1.

Remark 3.4: Compared with the existing results [5], [15], the newly developed method has the following advantages: (1) the nonlinear terms in the systems under consideration depend on both the fast and slow dynamics; (2) not only the existence of the stability bound is established, but also an estimate of the stability bound $\varepsilon_{0}$ is given.

Remark 3.5: [10], [23] have considered stability bound problem for a class of SPSs, where the nonlinear term $f(x)$ satisfies $\|f(x)\| \leq$ $\|F x\|$ with $F$ being a constant matrix. It can be seen that the sector condition (2) is a sub-class of the above mentioned nonlinearity. Lyapunov function (7) upon which Theorem 3.1 is based depends on the nonlinearities, and the design parameters $\lambda_{j}$ are not restricted to be positive, which provides an opportunity to reduce the conservatism of the proposed method. Thus it is expected that Theorem 3.1 can lead to a tighter stability bound than the existing methods [10], [23], as will be shown by the examples in Section IV.

Remark 3.6: The singular perturbation parameter $\varepsilon$ of SPSs is usually a positive number. Thus, the stability bound problem for SPSs is usually referred to as the problem of determining the stability bound $\varepsilon_{0}$ such that the system is stable for all $\varepsilon \in\left(0, \varepsilon_{0}\right)$ or $\left(0, \varepsilon_{0}\right]$. When $\varepsilon=0$, the SPS reduces to a Lur'e descriptor system whose stability problem has been considered by [16], [17]. From Theorem 2 of [17], system (1) is stable for all $\varepsilon \in\left[0, \varepsilon_{0}\right]$ if $C_{2} \Lambda=0$ and the conditions of Theorem 3.1 hold simultaneously.

Corollary 3.1: There is a scalar $\varepsilon_{0}>0$ such that system (1) is absolutely stable for any $\varepsilon \in\left(0, \varepsilon_{0}\right]$, if there exist a diagonal matrix $\Gamma>0$, matrices $Z_{i}(i=1,2,5)$ with $Z_{i}=Z_{i}^{T}(i=1,2)$ and $\Lambda=\operatorname{diag}\left\{\lambda_{1}, \lambda_{2}, \ldots, \lambda_{m}\right\}$ with $\lambda_{j}=0$ when $c_{1}^{j} \neq 0$ and $c_{2}^{j} \neq 0$, satisfying LMIs (21), (25) and

$$
Z_{2}>0 \text {. }
$$

Proof: If LMIs (21), (25) and (33) are feasible, there exists a sufficiently small scalar $\varepsilon_{0}>0$ and symmetric matrices $Z_{3}$ and $Z_{4}$ satisfying LMIs (22), (23) and (26). Then by Theorem 3.1, system (1) is absolutely stable for any $\varepsilon \in\left(0, \varepsilon_{0}\right]$.

The stability bound $\varepsilon_{0}$ given by Theorem 3.1 is guessed. Corollary 3.1 proposes sufficient conditions for the existence of the stability bound $\varepsilon_{0}$ which are also necessary conditions for the feasibility of the LMI conditions of Theorem 3.1. We now propose a bisectional search algorithm to get the best estimate for the stability bound $\varepsilon_{0}$.
Remark 3.7: In Algorithm 1, Step 1 presents the initial and terminal conditions. Steps 2-3 will show that the proposed method does not work or the optimal stability bound $\varepsilon_{0}$ is larger than the given $\gamma$ or determine a search interval $[\underline{\varepsilon}, \bar{\varepsilon}]$ for Steps 4-6 such that LMIs (21)-(23), (25) and (26) are feasible with $\varepsilon_{0}=\underline{\varepsilon}$ but not with $\varepsilon_{0}=\bar{\varepsilon}$. Steps 4-6 are used to search the best estimate of the stability bound $\varepsilon_{0}$ in $[\underline{\varepsilon}, \bar{\varepsilon}]$.

\section{EXAMPLES}

In this section, two examples are given to illustrate the proposed method and show its advantages over the existing results.

1) Example 1: Consider the singularly perturbed system

$$
\left\{\begin{array}{l}
\dot{x}_{1}=-2.75 x_{1}+4 x_{2}-3 x_{3}+4 x_{4}+0.25 \sin \left(x_{1}\right), \\
\dot{x}_{2}=2 x_{2}-x_{3}-2 x_{4}, \\
\varepsilon \dot{x}_{3}=x_{1}+2 x_{2}-1.75 x_{3}+3 x_{4}+0.25 \sin \left(x_{3}\right), \\
\varepsilon \dot{x}_{4}=2 x_{2}-3 x_{4} .
\end{array}\right.
$$

We first convert system (34) into the form of (4), where

$$
\begin{gathered}
E(\varepsilon)=\left[\begin{array}{llll}
1 & 0 & 0 & 0 \\
0 & 1 & 0 & 0 \\
0 & 0 & \varepsilon & 0 \\
0 & 0 & 0 & \varepsilon
\end{array}\right], A=\left[\begin{array}{cccc}
-3 & 4 & -3 & 4 \\
0 & 2 & -1 & -2 \\
1 & 2 & -2 & 3 \\
0 & 2 & 0 & -3
\end{array}\right], \\
B=\left[\begin{array}{cccc}
0.5 & 0 & 0 & 0 \\
0 & 0 & 0.5 & 0
\end{array}\right]^{T}, C=\left[\begin{array}{cccc}
1 & 0 & 0 & 0 \\
0 & 0 & 1 & 0
\end{array}\right]
\end{gathered}
$$

and $\phi=\left[\begin{array}{l}0.5\left(x_{1}+\sin \left(x_{1}\right)\right) \\ 0.5\left(x_{3}+\sin \left(x_{3}\right)\right)\end{array}\right]$. It can be seen that $\phi$ satisfies the sector condition (2) with $K=\left[\begin{array}{ll}1 & 0 \\ 0 & 1\end{array}\right]$.

Since the nonlinear function $\phi$ depends on both the fast and slow dynamics, the results of [15] can not be used to check the stability of the system. Meanwhile, the methods of [5] can not provide the stability bound $\varepsilon_{0}$ even if they are feasible.

Using Algorithm 1 with the aid of LMI Toolbox, we have $\varepsilon_{0}=$ 0.8139 for this system and the corresponding solution to the LMIs of Theorem 3.1 is as follows:

$$
\begin{aligned}
Z_{1} & =\left[\begin{array}{cc}
1343.238528 & -940.479823 \\
-940.479823 & 18218.447233
\end{array}\right], \\
Z_{2} & =\left[\begin{array}{ll}
3484.022542 & 2364.037454 \\
2364.037454 & 9762.215794
\end{array}\right], \\
Z_{3} & =\left[\begin{array}{cc}
-123.213474 & -1990.113104 \\
-1990.113104 & 6772.458016
\end{array}\right], \\
Z_{4} & =\left[\begin{array}{cc}
-1308.784981 & 2747.566762 \\
2747.566762 & 14505.272846
\end{array}\right], \\
Z_{5} & =\left[\begin{array}{cc}
936.951782 & -6947.220931 \\
2118.172105 & -14169.410321
\end{array}\right], \\
\Gamma & =\left[\begin{array}{cc}
284.076475 & 0 \\
0 & 1230.560102
\end{array}\right], \\
\Lambda & =\left[\begin{array}{cc}
241.825772 & 0 \\
0 & 464.455253
\end{array}\right] .
\end{aligned}
$$

$$
\begin{aligned}
\left.\dot{V}\right|_{(4)} & =\left[\begin{array}{l}
x \\
\phi
\end{array}\right]^{T}\left[\begin{array}{cc}
A^{T} Z(\varepsilon)+Z^{T}(\varepsilon) A & Z^{T}(\varepsilon) B+A^{T} C^{T} \Lambda \\
B^{T} Z(\varepsilon)+\Lambda C A & \Lambda C B+B^{T} C^{T} \Lambda
\end{array}\right]\left[\begin{array}{c}
x \\
\phi
\end{array}\right] \\
& =\left[\begin{array}{c}
x \\
K_{\Delta} C x
\end{array}\right]^{T}\left[\begin{array}{cc}
A^{T} Z(\varepsilon)+Z^{T}(\varepsilon) A & Z^{T}(\varepsilon) B+A^{T} C^{T} \Lambda \\
B^{T} Z(\varepsilon)+\Lambda C A & \Lambda C B+B^{T} C^{T} \Lambda
\end{array}\right]\left[\begin{array}{c}
x \\
K_{\Delta} C x
\end{array}\right] \\
& =x^{T}\left[\begin{array}{c}
I \\
K_{\Delta} C
\end{array}\right]^{T}\left[\begin{array}{cc}
A^{T} Z(\varepsilon)+Z^{T}(\varepsilon) A & Z^{T}(\varepsilon) B+A^{T} C^{T} \Lambda \\
B^{T} Z(\varepsilon)+\Lambda C A & \Lambda C B+B^{T} C^{T} \Lambda
\end{array}\right]\left[\begin{array}{c}
I \\
K_{\Delta} C
\end{array}\right] x \\
& =x^{T}\left[\left(A+B K_{\Delta} C\right)^{T}\left(Z(\varepsilon)+C^{T} \Lambda K_{\Delta} C\right)+\left(Z(\varepsilon)+C^{T} \Lambda K_{\Delta} C\right)^{T}\left(A+B K_{\Delta} C\right)\right] x .
\end{aligned}
$$


From Theorem 3.1, system (34) is absolutely stable for any $\varepsilon \in(0,0.8139]$.

Algorithm 1: Bisectional search algorithm determining $\varepsilon_{0}$.

Step 1. Step 1. Given positive scalars $\alpha, \beta, \gamma$ and $\delta$, where $\alpha$ and $\delta$ are sufficiently small, $\gamma$ is sufficiently large and $\alpha<\beta<\gamma$. Set $\underline{\varepsilon}=\bar{\varepsilon}=\beta$.

Step 2. Step 2. Check LMIs (21)-(23), (25) and (26) with $\varepsilon_{0}=\beta$. If they are feasible, set $\underline{\varepsilon}=\beta$ and $\beta:=2 \beta$; otherwise, set $\bar{\varepsilon}=\beta$ and $\beta:=0.5 \beta$.

Step 3. Step 3. If $\bar{\varepsilon}<\alpha$ or $\underline{\varepsilon}>\gamma$, go to Step 7. Else if $\underline{\varepsilon} \geq \bar{\varepsilon}$, go to Step 2.

Step 4. Step 4. Set $\varepsilon^{*}=0.5(\underline{\varepsilon}+\bar{\varepsilon})$.

Step 5. Step 5. Check LMIs (21)-(23), (25) and (26) with $\varepsilon_{0}=\varepsilon^{*}$. If they are feasible, set $\underline{\varepsilon}=\varepsilon^{*}$, otherwise, set $\bar{\varepsilon}=\varepsilon^{*}$.

Step 6. Step 6. Go to Step 4 if $|\underline{\varepsilon}-\bar{\varepsilon}|>\delta$, otherwise, go to Step 7 .

Step 7. Step 7. If $\bar{\varepsilon}<\alpha$, the proposed method can not give an answer. If $\underline{\varepsilon}>\gamma$, the optimal stability bound $\varepsilon_{0}$ is larger that $\gamma$. Otherwise, the optimal stability bound produced by the proposed method is the value of $\varepsilon_{0}$. End.

To show the advantage of the newly developed method, the authors make their effort to apply the method of [10] to system (34) and the obtained stability bound is 0.0923 , while the stability bound of system (34) computed by the method of [23] is 0.6541 . It can be seen that the stability bound of system (34) obtained by Theorem 3.1 is the least conservative.

2) Example 2: Consider an inverted pendulum controlled by a dc motor via a gear train whose physical model can be found in [24]. This system can be described by the following state equations:

$$
\left\{\begin{array}{l}
\dot{x}_{1}(t)=x_{2}(t), \\
\dot{x}_{2}(t)=\frac{g}{l} \sin x_{1}(t)+\frac{N K_{m}}{m l^{2}} x_{3}(t), \\
L_{a} \dot{x}_{3}(t)=-K_{b} N x_{2}(t)-R_{a} x_{3}(t)+u(t)
\end{array}\right.
$$

where $x_{1}(t)=\theta_{p}(t), x_{2}(t)=\dot{\theta}_{p}(t), x_{3}(t)=I_{a}(t), u(t)$ is the control input, $K_{m}$ is the motor torque constant, $K_{b}$ is the back emf constant, and $N$ is the gear ratio. The parameters for the plant are given as $g=9.8 \mathrm{~m} / \mathrm{s}^{2}, l=1 \mathrm{~m}, m=1 \mathrm{~kg}, N=10, K_{m}=0.1 \mathrm{Nm} / \mathrm{A}$, $K_{b}=0.1 \mathrm{Vs} / \mathrm{rad}, R_{a}=1 \Omega$ and $L_{a}=\varepsilon \mathrm{mH}$. Note that the inductance $L_{a}$ represents the small parameter in the system. Substituting the parameters into (35) and letting $u=-10 x_{1}-9 x_{2}$, we have

$$
\left\{\begin{array}{l}
\dot{x}_{1}(t)=x_{2}(t) \\
\dot{x}_{2}(t)=9.8 \sin x_{1}(t)+x_{3}(t), \\
\varepsilon \dot{x}_{3}(t)=-10 x_{1}-10 x_{2}(t)-x_{3}(t) .
\end{array}\right.
$$

System (36) can be transformed into the form of (4) with

$$
\begin{aligned}
E(\varepsilon) & =\left[\begin{array}{lll}
1 & 0 & 0 \\
0 & 1 & 0 \\
0 & 0 & \varepsilon
\end{array}\right], A=\left[\begin{array}{ccc}
0 & 1 & 0 \\
-9.8 & 0 & 1 \\
-10 & -10 & -1
\end{array}\right], \\
B & =\left[\begin{array}{lll}
0 & 9.8 & 0
\end{array}\right]^{T}, C=\left[\begin{array}{lll}
1 & 0 & 0
\end{array}\right]
\end{aligned}
$$

and $\phi=\sin \left(x_{1}\right)+x_{1}$ which satisfies (2) with $K=2$.

Using Algorithm 1 with the aid of LMI Toolbox, we get $\varepsilon_{0}=0.7145$ for this system and the corresponding solution to the LMIs of Theorem 3.1 is as follows:

$$
\begin{aligned}
Z_{1} & =\left[\begin{array}{cc}
424.713064 & 5.268733 \\
5.268733 & 18.589266
\end{array}\right], Z_{2}=2.9625077, \\
Z_{3} & =\left[\begin{array}{cc}
284.298125 & 19.533739 \\
19.533739 & -3.561747
\end{array}\right], Z_{4}=1.063497, \\
Z_{5} & =\left[\begin{array}{ll}
38.346446 & 2.113150
\end{array}\right], \\
\Gamma & =24.741906, \Lambda=-170.965352 .
\end{aligned}
$$

From Theorem 3.1, system (36) is absolutely stable for any $\varepsilon \in$ $(0,0.7145]$.

If we restrict $\Lambda$ to be positive, the stability bound computed by Algorithm 1 is 0.2743 which is smaller than 0.7145 . This shows the benefit of allowing $\Lambda$ to take negative values.

With the best effort, the authors found that the method of [10] is infeasible for system (36). Meanwhile, the stability bound of system (36) produced by the method of [23] is 0.0893 which is much smaller than 0.7145 .

The above numerical examples show that Theorem 3.1 leads to less conservatism than the methods obtained in [10], [23] if the nonlinearities satisfy the sector condition (2). The reason is that the Lyapunov function based on which Theorem 3.1 is derived is more general than those used in [10], [23].

\section{CONCLUSION}

In this technical note, we have considered absolute stability problem for Lur'e singularly perturbed systems whose nonlinear terms depend on both the fast and slow dynamics. We have constructed an $\varepsilon$-dependent Lur'e Lyapunov function and proposed an $\varepsilon$-independent LMI based stability criterion by which a bisectional search algorithm has been formulated to get the best estimate of the stability bound. The given examples have illustrated the effectiveness and advantages of the obtained method.

\section{REFERENCES}

[1] E. H. Abed, "A new parameter estimate in singular perturbations," Syst. Control Lett., vol. 6, no. 3, pp. 193-198, 1985.

[2] H. Khalil, "Stability analysis of nonlinear multiparameter singularly perturbed systems," IEEE Trans. Autom. Control, vol. AC-32, no. 3, pp. 260-263, Mar. 1987.

[3] B. S. Chen and C. L. Lin, "On the stability bounds of singularly perturbed systems," IEEE Trans. Autom. Control, vol. 35, no. 11, pp. 1265-1270, Nov. 1990.

[4] L. Saydy, "New stability/performance results for singularly perturbed systems," Automatica, vol. 32, no. 6, pp. 807-818, 1996.

[5] H. D. Tuan and S. Hosoe, "Multivariable circle criteria for multiparameter singularly perturbed systems," IEEE Trans. Autom. Control, vol. 45, no. 4, pp. 720-725, Apr. 2000.

[6] L. Cao and H. M. Schwartz, "Complementary results on the stability bounds of singularly perturbed systems," IEEE Trans. Autom. Control, vol. 49, no. 11, pp. 2017-2021, Nov. 2004.

[7] S. Sen and K. B. Datta, "Stability bounds of singularly perturbed systems," IEEE Trans. Autom. Control, vol. 38, no. 2, pp. 302-304, Feb. 1993.

[8] P. V. Kokotovic, H. K. Khalil, and J. O'Reilly, Singulurly Perturbation Methods in Control: Analysis and Design. New York: Academic, 1986.

[9] A. Saberi and H. Khalil, "Quadratic-type Lyapunov functions for singularly perturbed systems," IEEE Trans. Autom. Control, vol. AC-29, no. 6, pp. 542-550, Jun. 1984.

[10] Z. H. Shao, "Robust stability of two-time-scale systems with nonlinear uncertainties," IEEE Trans. Autom. Control, vol. 49, no. 2, pp. 258-261, Feb. 2004.

[11] H. Liu, F. Sun, and Z. Sun, "Stability analysis and synthesis of fuzzy singularly perturbed systems," IEEE Trans. Fuzzy Syst., vol. 13, no. 2 , pp. 273-284, Apr. 2005.

[12] G. H. Yang and J. X. Dong, "Control synthesis of singularly perturbed fuzzy systems," IEEE Trans. Fuzzy Syst., vol. 16, no. 3, pp. 615-629, Jun. 2008.

[13] H. K. Khalil, Nonlinear Systems, Third ed. Englewood Cliffs, NJ: Prentice-Hall, 1996.

[14] W. M. Haddad and D. S. Bernstein, "Explicit construction of quadratic Lyapunov functions for the small gain, positivity, circle and Popov theorems and their application to robust stability part i: Continous-time theory," Int. J. Robust Nonlin. Control, vol. 3, no. 4, pp. 313-339, 1993.

[15] V. R. Saksena and P. V. Kokotovic, "Singular perturbation of the Popov-Kalman-Yakubovich lemma," Syst. Control Lett., vol. 1, no. 1, pp. $65-68,1981$. 
[16] C. Yang, Q. Zhang, Y. Lin, and L. Zhou, "Positive realness and absolute stability problem of descriptor systems," IEEE Trans. Circuits Syst. I, vol. 54, no. 5, pp. 1142-1149, May 2007.

[17] C. Yang, Q. Zhang, and L. Zhou, "Strongly absolute stability of Lur'e descriptor systems: Popov-type criteria," Int. J. Robust Nonlin. Control, vol. 19, no. 7, pp. 786-806, 2009.

[18] C. Yang and Q. Zhang, "Multi-objective control for T-S fuzzy singularly perturbed systems," IEEE Trans. Fuzzy Syst., vol. 17, no. 1, pp. 104-115, Feb. 2009.

[19] W. M. Haddad and D. S. Bemstein, "Parameter-dependent Lyapunov functions and the Popov criterion in robust analysis and synthesis," IEEE Trans. Autom. Control, vol. 40, no. 3, pp. 536-543, Mar. 1995.

[20] V. A. Ugrinovskii and I. R. Petersen, "Guaranteed cost control of uncertain systems via Lur'e-postnikov Lyapunov functions," Automatica, vol. 36, no. 2, pp. 279-285, 2000.

[21] S. Boyd, L. E. Ghaoui, E. Feron, and V. Balakrishnan, Linear Matrix Inequalities in System and Control Theory. Philadelphia, PA: SIAM, 1994.

[22] P. Gahinet, A. Nemirovski, A. J. Laub, and M. Chilali, "LMI Control Toolbox," The Mathworks Inc., Tech. Rep., 1995.

[23] Y. Gao, G. Lu, and Z. Wang, "Passivity analysis of uncertain singularly perturbed systems," IEEE Trans. Circuits Syst. II, vol. 57, no. 6, pp. 486-490, Jun. 2010.

[24] S. H. Zak and C. A. Maccarley, "State-feedback control of non-linear systems," Int. J. Control, vol. 43, no. 5, pp. 1497-1514, 1986.

\section{Minimal Time Control of Fed-Batch Processes With Growth Functions Having Several Maxima}

\author{
Alain Rapaport and Denis Dochain
}

\begin{abstract}
We address the issue of minimal time optimal control of fedbatch reactor in presence of complex non monotonic kinetics. Several extremal paths with singular ares can be locally optimal. We show how a regularization technique can help determining the optimal synthesis, based on a numerical approach.
\end{abstract}

Index Terms-Fed-batch reactors, minimal time problem, optimal synthesis, regularization, singular arcs.

\section{INTRODUCTION}

Fed-batch bioreactors represent an important class of bioprocesses, mainly in the food industry (e.g. yeast production or wine making) and in the pharmaceutical industry (like the production of penicillin or of the vaccine against the Hepatitis B) but also e.g. for biopolymer applications (PHB) or for wastewater treatment (via Sequential Batch Reactors (SBR's) for instance). It is also very much involved in the field of enzyme production which has been developed over the past decade due to the recombinant DNA technology and via the use of filamentous micro-organisms. One of the key issues in the operation of fed-batch reactors is to optimize the process operation over a limited

Manuscript received September 11, 2010; revised December 09, 2010, December 10, 2010, and April 03, 2011; accepted May 11, 2011. Date of publication June 27, 2011; date of current version November 02, 2011. This work was supported in part by research results of the Belgian Programme on Inter-University Poles of Attraction initiated by the Belgian State, Prime Minister's office for Science, Technology and Culture. Recommended by Associate Editor R. D. Braatz.

A. Rapaport is with UMR INRA-SupAgro "MISTEA," Montpellier 34060, France and also with EPI INRA-INRIA "MODEMIC," Sophia-Antipolis, France (e-mail: rapaport@ supagro.inra.fr).

D. Dochain is with CESAME, Université catholique de Louvain, Louvain-laNeuve 1348, Belgium (e-mail: denis.dochain@uclouvain.be).

Digital Object Identifier 10.1109/TAC.2011.2159424 time period. An intensive research activity has been devoted to optimal control of (fed-batch) bioreactors mainly in the seventies and in the eighties (see, e.g., [12], [13]). In this work, we address the issue of minimal time optimal control of fed-batch reactor in presence of complex non monotonic kinetics, characterized here by the combination of two non-monotonic growth functions, aimed at emphasizing the presence of parallel metabolic pathways to transform the limiting substrate $S$ into the biomass $B$. It is well known that for such problems, the optimal synthesis is bang-bang with a possible singular arc in the presence of a single non-monotonic growth rate model [11]. In presence of combinations of several non-monotonic growths, the candidate singular arcs are multiple and determining which singular arc is eventually optimal is clearly a crucial issue. The case of batch chemical processes, with temperature as manipulated variable, has been extensively studied [1], [2]. Even though there are similarities between chemical and biological systems, the optimal control of the fed-batch bioprocess under consideration is somewhat different, at least with respect to the following two points. First of all, the control variable is the flow rate : this implies that the volume is an extra state variable, and the objective is to reach simultaneously precise values for both the volume and the substrate concentration. Secondly kinetics of fed-batch bioreactors are typically represented by non-monotonic functions of the reactants (with possibly more than one local maximum). The local optimality conditions based on the Pontryagin Maximum Principle allow to characterize the geometric structure of the extremal trajectories, in which there may be singular arcs [3]. These necessary conditions are not always sufficient for determining which extremals are (globally) optimal. One may look also for second-order conditions for determining the minimizer [4], [10], or has to compute the cost of each extremal or use global optimization methods such as dynamic programming or Hamilton-Jacobi-Bellman equation [18], [19]. The extremals are traditionally determined numerically by considering shooting methods (see for instance [18]). For bang-bang control, it is well known that one may face numerical troubles because the shooting function is in general not smooth [9]. Recently a smoothing method inspired from [15] has been proposed for the regularization of the shooting function [14]. The convergence of the method has been proven when the original problem admits a unique optimal solution. It has been also shown that the convergence of the control inputs is not guaranteed when the optimal solution presents a singular arc. For our problem, we first show that it is easy to determine analytically the singular arcs that are locally optimal, and that the determination of the optimal synthesis consists then in deciding which singular arc is optimal. We consider the regularization method used in [14], but in a different way. We do not apply a shooting method, that is relevant for given initial conditions; instead we fill the state space with extremals for the regularized problem, to proceed next to the study of the extremals. When extremals do not intersect, one can straightforwardly conclude about the optimal synthesis for the original problem (even though the convergence of the control inputs is not guaranteed). If extremals intersect, one may distinguish sub-domains of the state space for which it is possible to conclude about the optimal synthesis. We believe that this technique is particularly efficient for planar dynamics for which it is easy to visualize this field. In our problem, the multiplicity of the singular arcs reveals the existence of a locus for which several extremals have the same cost. The contribution of the technical note is two-fold. We first propose a proof based on differential inclusions arguments that allows to relax the assumption of the uniqueness of the optimal solution for the convergence of the optimal paths (Section IV). Then we show how to apply numerically the approximation procedure for analyzing the field of extremals on the whole state space (Section V). 